\title{
Téoros
}

Revue de recherche en tourisme

\section{Tourisme et restauration : des liens qui se resserrent}

\section{Louis Jolin}

Volume 3, numéro 3, novembre 1984

Cuisine québécoise, restauration et tourisme : vers une synergie

féconde

URI : https://id.erudit.org/iderudit/1080772ar

DOI : https://doi.org/10.7202/1080772ar

Aller au sommaire du numéro

Éditeur(s)

Université du Québec à Montréal

ISSN

0712-8657 (imprimé)

1923-2705 (numérique)

Découvrir la revue

Citer ce document

Jolin, L. (1984). Tourisme et restauration : des liens qui se resserrent. Téoros, 3(3), 1-1. https://doi.org/10.7202/1080772ar d'utilisation que vous pouvez consulter en ligne.

https://apropos.erudit.org/fr/usagers/politique-dutilisation/ 


\section{Présentation}

\section{Tourisme et restauration: des liens qui se resserrent}

Grâce à la collaboration étroite du Centre de recherches technologiques de I'I.T.H.Q, et de son directeur, monsieur Jean Gaudreau, la revue Téoros offre son menu d'automne, un numéro consacré spécialement à la restauration. Certes, il s'agit là d'un sujet ambitieux et épineux surtout que les problèmes de la restauration au Québec sont fort nombreux. Depuis plusieurs années I'Association des restaurateurs du Québec s'inquiète de la proliferation des restaurants au Québec, déplore la diminution des profits et le nombre de faillites annuellement, réclame des mesures gouvernementales qui auraient pour effet de relancer l'industrie de la restauration: suppression de la taxe supplémentaire sur le vin pour les restaurateurs, remplacement de la taxe actuelle sur les repas de plus de $3.25 \$$ par une taxe de 5 pour cent sur tous les repas quelque soit le prix, etc...

Ces problèmes sont connus et maintes fois énoncés. Certains d'entre eux sont évoqués de nouveau dans l'un ou l'autre des articles, quelques solutions possibles sont suggérées mais tout cela ne constitue pas l'essentiel du numéro. Compte tenu de l'orientation de Téoros, une autre approche a été privilégiée. En effet la restauration $y$ est abordée plutôt sous /'angle de ses liens avec l'expérience touristique dans son ensemble, l'industrie de la restauration y est vue comme une composante importante de l'industrie touristique.

Plusieurs auteurs, recrutés pour plusieurs parmi le corps professoral de l'Institut de tourisme et d'hötellerie du Québec, ont mis leur talent à cerner les liens entre le tourisme et la restauration tout en n'oubliant pas que le phénomène de la restauration, au Québec comme ailleurs dans le monde, est en perpétuelle évolution. Le tourisme et la gastronomie sont mis en relation dialectique comme le sont les voyages $d^{\prime} U / y s s e$ et d'Urien, la restauration québécoise est scrutée dans ses structures, dans ses mouvements d'intégration horizontale et verticale et dans ses rapports obligés avec une clientèle aux aspirations et aux besoins divers. La restauration, pour constituer une des composantes de l'offre touristique, doit s'appuyer sur une cuisine affirmée dans ses caractéristiques qu'elle soit traditionnelle ou nouvelle; des articles rappelent donc l'histoire de la cuisine québécoise, de Jacques Cartier à nos jours, et soulignent /'essor relativement récent de la nouvelle cuisine.
Certaines expériences concrètes - tables champêtres, développement d'une gastronomie typique en Estrie - tracent des voies nouvelles dans le sens d'une affirmation de la cuisine régionale comme attrait touristique. Les habitudes et les comportements de la clientele locale ou touristique sont en mutation, les découvertes technologiques ouvrent de nouvelles perspectives, la restauration des années 80 ne sera peut-être pas celle de l'an 2000. II faut s'y préparer. La formation des ressources humaines entre dans cette préparation, mais intègre-telle suffisamment la dimension touristique?

Autant d'affirmations, autant d'interrogations au menu de ce numéro spécial qui, je l'espère, aura su vous mettre en appétit. $\dot{A}$ vous maintenant d'en déguster les plats et $n^{\prime}$ hésitez pas à nous faire part de vos réflexions, de vos commentaires, de vos critiques. Téoros est un lien entre les milieux de la recherche et de l'enseignement et les professionne/s du tourisme... il ne doit pas fonctionner à sens unique.

Enfin le comité de rédaction de Téoros remercie sincèrement le Centre de recherches technologiques et les professeurs de I'I. T. H.Q. pour leur participation à la confection de ce numéro, participation qui s'inscrit dans cette synergie tant de fois souhaitée des divers intervenants en tourisme.

Louis Jolin

Directeur de Téoros 